\title{
Measurement of Knee Rotation Angles Using a Smartphone Application: An Experimental Study of Porcine Knees
}

\author{
Hee-June Kim, MD ${ }^{1}$, Hyun-Joo Lee, $\mathrm{MD}^{1}$, Ji-Yeon Shin, $\mathrm{MD}^{2}$, Young-Seo Choi, $\mathrm{MD}^{1}$, and Hee-Soo Kyung, $\mathrm{MD}^{1}$ \\ ${ }^{1}$ Department of Orthopaedic Surgery, Kyungpook National University Hospital, Daegu; ${ }^{2}$ Department of Preventive Medicine, Eulji University School of Medicine, \\ Daejeon, Korea
}

Purpose: This study evaluated the efficacy of a smartphone application in the measurement of rotation angles in porcine knees.

Materials and Methods: Two K-wires were fixated to the femoral condyle and anterior tibial crest of 10 porcine legs. The angle created between the $\mathrm{K}$-wires with an external rotation force applied was measured on a photograph and defined as the true angle. The same force was applied to the legs placed on a splint with a smartphone attached to the plantar side. The angle presented on a smartphone application was determined as the measured angle. The differences between the true and measured angles in $30^{\circ}$ and $90^{\circ}$ knee flexion and differences in measured angles depending on the status of the popliteus tendon were compared.

Results: In the intact knees, the mean true angles in $30^{\circ}$ and $90^{\circ}$ flexion were $20.5^{\circ} \pm 1.4^{\circ}$ and $19.1^{\circ} \pm 1.3^{\circ}$, respectively, and the mean measured angles in $30^{\circ}$ and $90^{\circ}$ flexion were $21.1^{\circ} \pm 0.9^{\circ}$ and $18.6^{\circ} \pm 1.6^{\circ}$, respectively. When the popliteus tendon was cut, the mean true angles in $30^{\circ}$ and $90^{\circ}$ flexion were $31.4^{\circ} \pm 1.1^{\circ}$ and $38.5^{\circ} \pm 2.5^{\circ}$, respectively, and the mean measured angles in $30^{\circ}$ and $90^{\circ}$ flexion were $31.8^{\circ} \pm 1.2^{\circ}$ and $39.2^{\circ} \pm 2.8^{\circ}$, respectively. The differences between the true and measured angles were not significant. The measured angle increased by more than $10^{\circ}$ after cutting of the popliteus tendon in both $30^{\circ}$ and $90^{\circ}$ flexion.

Conclusions: Using a smartphone application could be a good method of measuring knee rotation.

Keywords: Knee, Rotation, Smartphone, Mobile application

\section{Introduction}

There are several types of rotatory instability of the knee joint including posterolateral rotatory instability (PLRI) due to posterolateral corner (PLC) injury, internal tibial rotatory instability due to anterolateral ligament (ALL) injury, and anteromedial instability. The obliquity of a reconstructed anterior cruciate ligament (ACL) could also affect rotatory instability and long-term

Received June 30, 2017; Revised August 1, 2017;

Accepted August 21, 2017

Correspondence to: Hee-Soo Kyung, MD

Department of Orthopaedic Surgery, Kyungpook National University

Hospital, 130 Dongdeok-ro, Jung-gu, Daegu 41944, Korea

Tel: +82-53-200-5636, Fax: +82-53-422-6605

E-mail: hskyung@knu.ac.kr

This is an Open Access article distributed under the terms of the Creative Commons Attribution Non-Commercial License (http://creativecommons.org/licenses/by-nc/4.0/) which permits unrestricted non-commercial use, distribution, and reproduction in any medium, provided the original work is properly cited. outcome $e^{1)}$. Therefore, the measurement of rotation is important to evaluate these problems of the knee joint.

Several methods are used to measure rotatory instability. The dial test was popularized in evaluating $\mathrm{PLRI}^{2)}$; several authors used rotational knee laxity measurement devices, such as ROTAM (Genourob, Laval, France), Lars Rotational Laxiometer (Lars Inc., Dijon, France), or a custom-made isotonic rotation machine ${ }^{3-5)}$. The dial test is also an easy and simple method used to assess injuries to the knee; however, it has limitations in terms of reproducibility and inter-observer reliability caused by variability in the angle based on the views of the observer. The knee laxity measurement devices are advantageous for accurate measurement, but it has disadvantages of bulky size and high cost.

Many smartphones are equipped with various sensors, including accelerometers, light sensors, temperature sensors, gyroscopes, barometers, etc. These sensors have become an abundant data source in measuring various aspects of a user's daily life. In particular, a gyroscope measures a smartphone's rotation rate by 
detecting the roll, pitch, and yaw motions of the phone along the $\mathrm{x}-$, $\mathrm{y}-$, and $\mathrm{z}$-axes, respectively; it is helpful in navigation applications as well as some smartphone games that use rotation data. In activity recognition research, a gyroscope is used to assist in mobile orientation detection ${ }^{6}$.

Therefore, we hypothesized that an application using the gyroscope sensor could be used in measuring the rotation angle of the knee. This study evaluated the rotation angles of porcine knees using a smartphone application and compared the differences with measurement using photographs.

\section{Materials and Methods}

\section{Measurement of True Rotation Angle}

A total of 10 porcine legs were used, and the specimens were disarticulated at the hip and ankle joints. All pigs weighed over 90 $\mathrm{kg}$, were at least 8 months old, and had no disease. First, two Kwires were fixated to the femoral condyle along the long axis and anterior tibial crest; the angle between the two wires was set to be $0^{\circ}$ when viewing along the long axis. The tibia was externally rotated, and a photograph was obtained along the long axis. The ImageJ software (National Institutes of Health, Bethesda, MD, USA) was used to measure the angle between the two K-wires, which was determined as the true angle (Fig. 1).

\section{Measurement of Rotation Angle Using a Smartphone}

\section{Application}

Measurement using a smartphone application was performed as follows. A short leg splint was placed under the specimen, and a smartphone was attached to the plantar side of the splint. The Rotating Sphere Inclinometer ver. 1.6 (Calmatics, Goteborg, Sweden), which can measure the roll and pitch and lateral longitudinal inclination, was used. The tibia was rotated externally, and the rotation angle presented on the application was measured. This angle was determined as the measured angle (Fig. 2).

\section{Rotation Measurement before and after Cutting of the Popliteus Tendon}

The true angle on the photographs and the measured angle on the smartphone application were obtained. Each angle was obtained in both $30^{\circ}$ and $90^{\circ}$ knee flexion before popliteus tendon cutting. After cutting of the popliteus tendon of the porcine knees, each angle was obtained again with the same method used before popliteus tendon cutting. The differences between the true angle and measured angle and differences in the measured angles depending on the status of the popliteus tendon were compared.

\section{Statistical Analysis}

We conducted a paired $t$-test to compare the true angle with the measured angle and the measured angles before and after cutting the popliteus tendon. We also estimated interdevice (interscorer) reliability using the intraclass correlation coefficient (ICC) (twoway random single measures). All statistical analyses were performed using the IBM SPSS ver. 19.0 (IBM Co., Armonk, NY, USA), and a p-value of $<0.05$ was considered statistically significant.
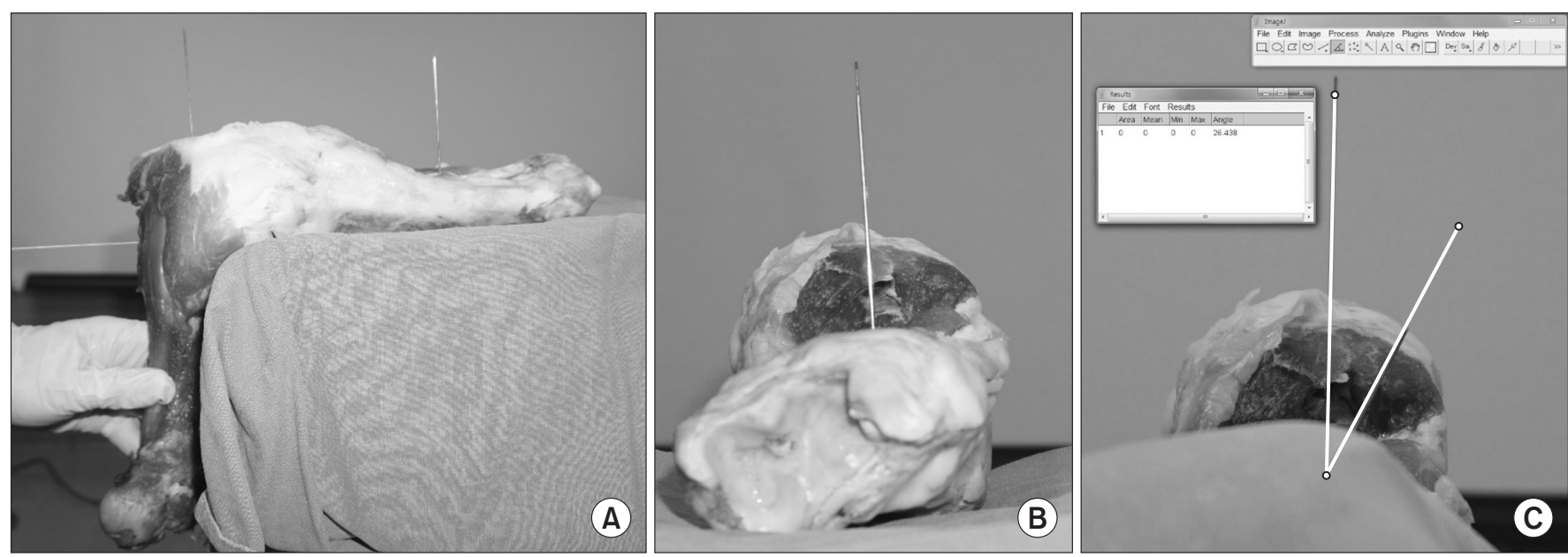

Fig. 1. Measurement of the true angle. (A) The K-wires are fixed to the femoral condyle along the long axis and anterior tibial crest. (B) The angle between the two K-wires is set to be $0^{\circ}$ when viewed along the long axis. (C) The tibia is externally rotated, and the photograph is obtained along the long axis. The angle between the two K-wires is measured using the imageJ software, and it is determined as the true angle. 

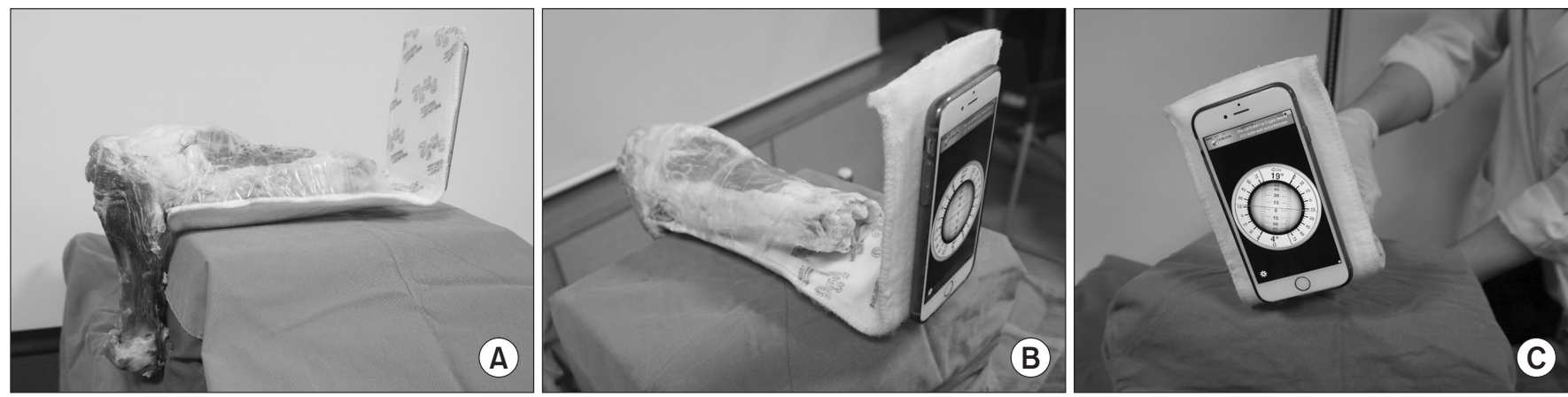

Fig. 2. Measurement of the rotation angle using a smartphone application. (A) A short leg splint is placed under the porcine knee. (B) The smartphone is attached to the plantar side of the splint. (C) The tibia is rotated externally, and the rotating angle presented on the application is measured.

Table 1. Results of the Dial Test before and after Popliteus Tendon Cutting

\begin{tabular}{|c|c|c|c|c|}
\hline Flexion $\left(^{\circ}\right)$ & $\begin{array}{c}\text { True } \\
\text { angle }\left(^{\circ}\right)\end{array}$ & $\begin{array}{c}\text { Measured } \\
\text { angle }\left(^{\circ}\right)\end{array}$ & $\mathrm{p}$-value & ICC \\
\hline \multicolumn{5}{|c|}{ Before popliteus cutting } \\
\hline 30 & $20.5 \pm 1.4$ & $21.1 \pm 0.9$ & 0.14 & 0.748 \\
\hline 90 & $19.1 \pm 1.3$ & $18.6 \pm 1.6$ & 0.23 & 0.773 \\
\hline \multicolumn{5}{|c|}{ After popliteus cutting } \\
\hline 30 & $31.4 \pm 1.1$ & $31.8 \pm 1.2$ & 0.21 & 0.837 \\
\hline 90 & $38.5 \pm 2.5$ & $39.2 \pm 2.8$ & 0.09 & 0.944 \\
\hline
\end{tabular}

Values are presented as mean \pm standard deviation.

ICC: intraclass correlation coefficient.

\section{Results}

In the porcine knees with an intact popliteus tendon, the mean true angle was $20.5^{\circ} \pm 1.4^{\circ}$ and the mean measured angle was $21.1^{\circ} \pm 0.9^{\circ}$ in $30^{\circ}$ knee flexion ( $\mathrm{p}=0.14$ ). The mean true angle was $19.1^{\circ} \pm 1.3^{\circ}$ and the mean measured angle was $18.6^{\circ} \pm 1.6^{\circ}$ in $90^{\circ}$ knee flexion ( $\mathrm{p}=0.23$ ). After cutting of the popliteus tendon, the mean true angle was $31.4^{\circ} \pm 1.1^{\circ}$ and the mean measured angle was $31.8^{\circ} \pm 1.2^{\circ}$ in $30^{\circ}$ knee flexion ( $\mathrm{p}=0.21$ ); further, the mean true angle was $38.5^{\circ} \pm 2.5^{\circ}$ and the mean measured angle was $39.2^{\circ} \pm 2.8^{\circ}$ in $90^{\circ}$ knee flexion ( $\mathrm{p}=0.09$ ). The differences between the true angle and measured angle were not significant and the agreement between the two assessments was excellent (ICC, 0.773 to 0.944 ) (Table 1).

The measured angle increased by more than $10^{\circ}$ after cutting of the popliteus tendon in both $30^{\circ}$ and $90^{\circ}$ flexion $(\mathrm{p}<0.001)$.

\section{Discussion}

In this study, the rotation angle of the porcine knees could be obtained and digitalized easily using the smartphone application, and the obtained values were not significantly different from those obtained using photographs.

The main stabilizing structures of the PLC of the knee joint are composed of the lateral collateral ligament, popliteus tendon, and popliteofibular ligament ${ }^{7-9}$. Injuries to the PLC can cause PLRI of the knee joint, which is often accompanied by ACL or posterior cruciate ligament (PCL) tears ${ }^{10,11}$. Untreated PLC injuries are responsible for the failure of ACL and PCL reconstructions ${ }^{2,12,13}$; therefore, the diagnosis of PLC injury is important for the treatment of knee injury.

The dial test is commonly used to diagnose PCL and PLC injuries. This test was performed in $30^{\circ}$ and $90^{\circ}$ of knee flexion to measure the angle between the thigh and foot. If the external rotation of an injured tibia exceeds $10^{\circ}$ in a side-to-side comparison with the contralateral tibia, an injury to the PLC is suggested. An increased external rotation at only $30^{\circ}$ indicates an isolated injury to the PLC, whereas an increased external rotation at both $30^{\circ}$ and $90^{\circ}$ suggests injury to both the PCL and PLC ${ }^{4,5,14,15)}$. This test is an easy and simple method used to assess the injury. Thighfoot angles can be obtained using the photographs or a goniometer while maintaining the tibial rotation ${ }^{5}$. However, this method has a disadvantage of inducing variability according to the views of the observer. Machines measuring rotational knee laxity could be useful, and accurate rotation has been reported by several authors. However, discomfort in moving the machine and high cost are some disadvantages associated with the use of such devices. By contrast, smartphones, as we used in this study, have become popularized, and no cost is involved in the installation of applications. Rotation measurement was easy by simply attaching it to the splint and reading the angle of the smartphone. Therefore, the effectiveness of the dial test using a smartphone application could be verified. Considering the no installation cost, high reproducibility, easy handling, and digitalization, using a smartphone application could be a good method of measuring rotation of the 

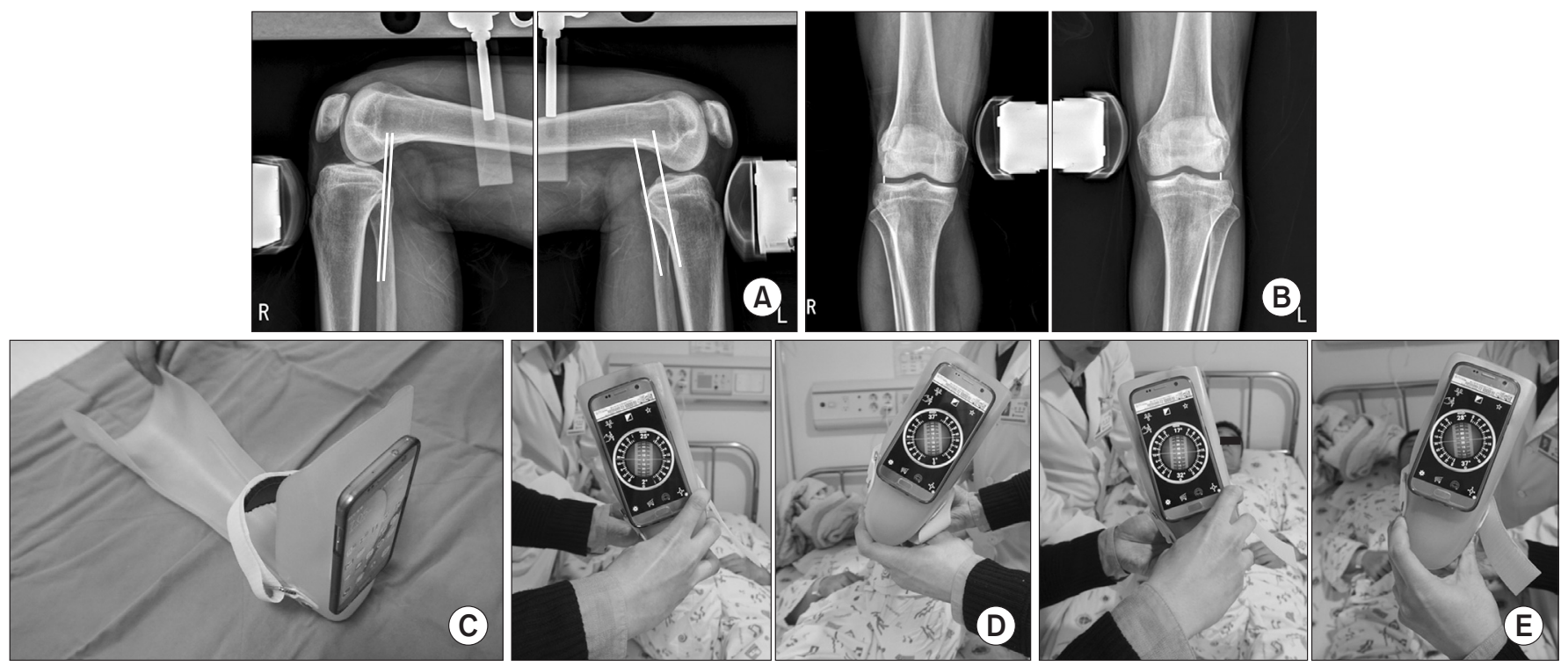

Fig. 3. Measurement of the rotation angle in patients with posterior cruciate ligament and posterolateral corner injuries in the left knee. (A) Posterior stress radiographs showing a side-to-side difference of $13 \mathrm{~mm}$. (B) Lateral stress radiographs showing a side-to-side difference of $2 \mathrm{~mm}$. (C) A custom-made instrument for measuring the rotation angle. (D) The rotation angle at $30^{\circ}$ flexion was $17^{\circ}$ in the right knee and $28^{\circ}$ in the left knee. (E) The rotation angle at $90^{\circ}$ flexion was $25^{\circ}$ in the right knee and $37^{\circ}$ in the left knee.

knee joint.

Several authors used animal models for human knee research, including dogs, cows, sheep, goats, and pigs ${ }^{16)}$. Among those, the porcine knees models were similar anatomically, radiographically, and arthroscopically to the human knees ${ }^{17}$. In this study, the popliteus tendon could be found in the porcine model; therefore, we could simulate the popliteus tendon injury by cutting the popliteus tendon in the femoral attachment site.

The smartphone application method was comparable to the method using photographs in rotation measurement. It has advantages of easy digitalization and handling. Moreover, it requires no installation cost and has good reproducibility. Therefore, it would be applicable clinically as a method of measuring rotation conveniently in humans with rotatory instability resulting from injuries of the PLC or ALL (Fig. 3).

This study has several limitations. The number of cases was small. External rotation was not performed by a constant force or torque, and it was performed until the examiner could feel the end-point of external rotation. However, we made an effort to apply the same amount of force during measurement of both the true and measured angles. Finally, this study did not account for differences between human knees and porcine knees, and the function of the popliteus tendon in porcine knees may not be similar to that in human knees. However, the objective of this study was to evaluate the effectiveness of a smartphone application in measurement of rotation angles.

\section{Conclusions}

Using a smartphone application could be a useful method for measuring knee rotation angle, which could be applicable with ease in patients with rotatory instability.

\section{Conflict of Interest}

No potential conflict of interest relevant to this article was reported.

\section{References}

1. Jeon YS, Choi SW, Park JH, Yoon JS, Shin JS, Kim MK. Midterm outcomes of anterior cruciate ligament reconstruction with far anteromedial portal technique. Knee Surg Relat Res. 2017;29:19-25.

2. Noyes FR, Barber-Westin SD, Roberts CS. Use of allografts after failed treatment of rupture of the anterior cruciate ligament. J Bone Joint Surg Am. 1994;76:1019-31.

3. Ruiz N, Filippi GJ, Gagniere B, Bowen M, Robert HE. The Comparative role of the anterior cruciate ligament and anterolateral structures in controlling passive internal rotation of the knee: a biomechanical study. Arthroscopy. 2016;32: 1053-62.

4. Bleday RM, Fanelli GC, Giannotti BF, Edson CJ, Barrett TA. 


\section{Kim et al. Knee Rotation Measurement Using a Smartphone Application}

Instrumented measurement of the posterolateral corner. Arthroscopy. 1998;14:489-94.

5. Bae JH, Choi IC, Suh SW, Lim HC, Bae TS, Nha KW, Wang $\mathrm{JH}$. Evaluation of the reliability of the dial test for posterolateral rotatory instability: a cadaveric study using an isotonic rotation machine. Arthroscopy. 2008;24:593-8.

6. Su X., Tong H., Ji P. Activity recognition with smartphone sensors. Tsinghua Sci Technol. 2014;19:235-49.

7. Terry GC, LaPrade RF. The posterolateral aspect of the knee: anatomy and surgical approach. Am J Sports Med. 1996;24: 732-9.

8. Brinkman JM, Schwering PJ, Blankevoort L, Kooloos JG, Luites J, Wymenga AB. The insertion geometry of the posterolateral corner of the knee. J Bone Joint Surg Br. 2005;87: 1364-8.

9. LaPrade RF, Morgan PM, Wentorf FA, Johansen S, Engebretsen L. The anatomy of the posterior aspect of the knee. An anatomic study. J Bone Joint Surg Am. 2007;89:758-64.

10. Djian P. Posterolateral knee reconstruction. Orthop Traumatol Surg Res. 2015;101(1 Suppl):S159-70.

11. Yoon KH, Lee SH, Park SY, Park SE, Tak DH. Comparison of anatomic posterolateral knee reconstruction using 2 different popliteofibular ligament techniques. Am J Sports Med. 2016;44:916-21.

12. Harner CD, Vogrin TM, Hoher J, Ma CB, Woo SL. Biomechanical analysis of a posterior cruciate ligament reconstruction: deficiency of the posterolateral structures as a cause of graft failure. Am J Sports Med. 2000;28:32-9.

13. LaPrade RF, Resig S, Wentorf F, Lewis JL. The effects of grade III posterolateral knee complex injuries on anterior cruciate ligament graft force: a biomechanical analysis. Am J Sports Med. 1999;27:469-75.

14. Noyes FR, Stowers SF, Grood ES, Cummings J, VanGinkel LA. Posterior subluxations of the medial and lateral tibiofemoral compartments: an in vitro ligament sectioning study in cadaveric knees. Am J Sports Med. 1993;21:407-14.

15. Loomer RL. A test for knee posterolateral rotatory instability. Clin Orthop Relat Res. 1991;(264):235-8.

16. Meyer RD, Tamarapalli JR, Lemons JE. Arthroscopy training using a "black box" technique. Arthroscopy. 1993;9:338-40.

17. Voto SJ, Clark RN, Zuelzer WA. Arthroscopic training using pig knee joints. Clin Orthop Relat Res. 1988;(226):134-7. 